\title{
Factors associated with good TB infection control practices among primary healthcare workers in the Free State Province, South Africa
}

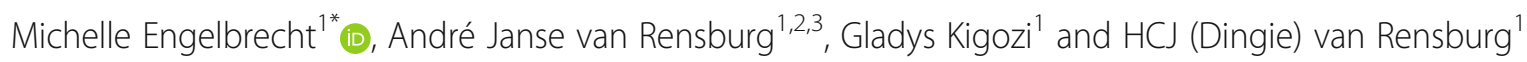

\begin{abstract}
Background: Despite the availability of TB infection control guidelines, and good levels of healthcare worker knowledge about infection control, often these measures are not well implemented. This study sought to determine the factors associated with healthcare workers' good TB infection control practices in primary health care facilities in the Free State Province, South Africa.

Methods: A cross-sectional self-administered survey among nurses $(n=202)$ and facility-based community healthcare workers $(n=34)$ as well as facility observations were undertaken at all 41 primary health care facilities in a selected district of the Free State Province.

Results: The majority of respondents were female $(n=200 ; 87.7 \%)$ and the average age was 44.19 years (standard deviation \pm 10.82$)$. Good levels of knowledge were recorded, with $42.8 \%(n=101)$ having an average score (i.e. $65-$ $79 \%)$ and $31.8 \%(n=75)$ a good score (i.e. $\geq 80 \%)$. Most respondents $(n=189 ; 80.4 \%)$ had positive attitudes towards TB infection control practices (i.e. $\geq 80 \%)$. While good TB infection control practices were reported by $72.9 \%(n=161)$ of the respondents (i.e. $\geq 75 \%$ ), observations revealed this to not necessarily be the case. For every unit increase in attitudes, good practices increased 1.090 times (Cl:1.016-1.169). Respondents with high levels of knowledge ( $\geq 80 \%)$ were 4.029 (Cl: 1.550-10.469) times more likely to have good practices when compared to respondents with poor levels of knowledge (<65\%). The study did not find TB/HIV-related training to be a predictor of good practices.

Conclusions: Positive attitudes and good levels of knowledge regarding TB infection control were the main factors associated with good infection control practices. Although many respondents reported good infection control practices - which was somewhat countered by the observations - there are areas that require attention, particularly those related to administrative controls and the use of personal protective equipment.
\end{abstract}

Keywords: TB infection control, Knowledge, Attitudes, Practices, Primary health care, Healthcare workers

\section{Background}

Healthcare associated infections (HAIs) are responsible for a substantial burden of diseases among patients and healthcare workers, especially in resource-poor settings [1]. The World Health Organization (WHO) reports that at any given time the prevalence of HAIs in developed countries varies between 3.5 and $12 \%$, and in developing

\footnotetext{
*Correspondence: engelmc@ufs.ac.za

${ }^{1}$ Centre for Health Systems Research \& Development, University of the Free

State, Nelson Mandela Road, Bloemfontein 9300, South Africa

Full list of author information is available at the end of the article
}

countries between 5.7 and $19.1 \%$ [2]. Of particular concern in high burdened Mycobacterium tuberculosis (TB) and human immunodeficiency virus (HIV) settings, is the nosocomial spread of TB among patients and healthcare workers, as a result of poor airborne infection control procedures, and further aggravated by the HIV epidemic as well as the emergence of multi-drug (MDR) and extensively drug resistant (XDR) TB [1, 3-5].

South Africa is classified as a high TB and HIV burdened country, with a TB incidence of 834 cases per 100000 population [6] and an HIV prevalence among 
adults aged 15-49 years of $17.9 \%$ [7]. International headlines were made in 2006, with the outbreak of XDR TB in Tugela Ferry, a rural community in South Africa. It was found that in all likelihood the transmission of the XDR TB strain occurred nosocomially [8]. The TB and HIV epidemics are intricately intertwined: approximately $61 \%$ of TB cases are co-infected with HIV and TB mortality among HIV-positive people was estimated to be 134 per 100000 population [6]. Immunecompromised people are more likely to develop active TB than those with normal immunity levels, i.e. 50$60 \%$ of HIV positive people will develop active disease. The annual risk of TB in an HIV positive person is $10 \%$ compared to a lifetime risk of $10 \%$ in a healthy individual [9].

The dual burden of TB and HIV also severely impacts South African healthcare workers. Occupational exposure to TB is a major health risk for healthcare workers healthcare workers are three times more likely to acquire TB than the general population $[10,11]$ and an estimated $81 \%$ of TB cases among healthcare workers are attributable to exposure in healthcare settings [12]. Furthermore, the high prevalence of latent TB Infection (LTBI) among healthcare workers [13] (84 \%, $69 \%$ and $62 \%$ using the tuberculin skin test, QuantiFERON-TB Gold-In-Tube and T-SPOT.TB, respectively), especially when compared to the general population, also points to TB being an occupationally acquired disease [14]. The HIV epidemic equally affects healthcare workers, with estimates of the HIV prevalence among healthcare workers ranging from 11.5 to $20.0 \%$ [15].

As TB transmission frequently occurs before an accurate diagnosis is made, it is the responsibility of healthcare workers, particularly managers, to ensure the implementation of appropriate TB infection control measures in all high risk settings so as to ensure that "health care facilities become known as places of healing and safety, not death and contagion" [16]. Good TB infection control relies on the early identification, isolation and rapid initiation of effective treatment of TB suspects combined with good organisation in facilities to avoid overcrowding and ensure appropriate patient flow [10]. More specifically, interventions promoting TB infection control practices in healthcare settings include improving: cough etiquette, respiratory hygiene and natural ventilation in waiting areas and consultation rooms; systematic and regular screening of healthcare workers for TB; and Isoniazid preventative therapy (IPT) and antiretroviral treatment (ART) for HIV positive patients and healthcare workers $[1,10]$. South African National TB management guidelines [9], specifically refer to healthcare workers' increased risk of TB and provide strategies to reduce their exposure to infection: 1) informing healthcare workers about signs and symptoms of TB and encouraging early screening and testing; 2) classifying healthcare workers with signs and symptoms of TB as "high risk TB suspects"; 3) providing voluntary HIV counselling and testing and encouraging healthcare workers to know their HIV status; 4) advocating/providing precautionary measures for HIV positive staff, such as IPT and ART; and 5) appropriate placement of HIV positive staff in low TB risk areas of the facility.

Notwithstanding the availability of guidelines on the implementation of $\mathrm{TB}$ infection control practices in healthcare facilities [17, 18], research conducted in South African health facilities found that infection control is often not well implemented [19-27] despite healthcare workers displaying good levels of knowledge about infection control practices [19, 20, 25]. While knowledge may not always be associated with good practices, research has found that motivation and support are priorities for the implementation of interventions to increase healthcare workers' appropriate execution of TB infection control practices [20].

Very little research currently exists that explores the factors that promote good infection control practices among healthcare workers. This gap is especially pressing at the primary health care (PHC) level, the main point of entry for state-supported TB patient care in many low-and middle-income settings such as South Africa. Healthcare workers often do not have the capacity and resources to correctly interpret, apply and manage policy directives in local contexts. Infection control is dependent on the hierarchy of measures (i.e. facility, administrative and environmental controls as well as personal protective equipment) [17] that together reduce the risk of spreading TB. Research has found that failed facility, administrative and environmental controls means that the focus is on personal protective measure (i.e. the use of N95 respirators), which shifts the burden of managing infection control onto the individual healthcare worker [24]. Accordingly, this study sought to determine the factors associated with healthcare workers' good TB infection control practices in PHC facilities.

\section{Methods \\ Setting}

The Free State, one of the nine provinces in South Africa, is situated in the centre of the country. With a total area of 129825 square kilometers, the Free State is the country's third-largest province, with a population of 2753200 people. The selected district covers 6284 square kilometers and is home to 747431 inhabitants [28]. In 2012, the TB incidence in the Free State was 708.5 cases per 100000 population, which was higher than the incidence in the country as a whole - 687.3 cases per 100100 [29]. The HIV prevalence was also 
higher in the Free State at $19.6 \%$ (15-49 years old) than the national prevalence of $17.9 \%$ [7]. Previous indications are that TB infection control is sub-optimal in some public health facilities in this region $[26,30]$, underlining the need to determine what influences good infection control practices.

\section{Design and population}

A cross sectional survey as well as facility observations were undertaken at all $41 \mathrm{PHC}$ facilities located in a selected district of the Free State Province, South Africa. Self-administered questionnaires were distributed to all categories of nurses and facility-based community healthcare workers (CHCWs) employed at these facilities.

\section{Instrument development, data collection and analysis}

The survey questionnaire comprising of four sections demographic information and knowledge, attitudes and practices (KAP) scales related to TB prevention and infection control-was developed based on instruments used in similar studies [20, 21, 25, 31-35]. The knowledge scale contained 22 statements with the response categories "True", "False" or "I don't know". The scale investigated knowledge about: TB symptoms; transmission; personal protective equipment; cough etiquette; ventilation; separation of coughing patients; and $\mathrm{TB} /$ HIV. The attitude scale comprised 18 statements with the response categories "Strongly agree", "Agree", "Disagree", or "Strongly disagree". Topics covered in the scale included: TB/HIV; training; fear of contagion; infection control; personal protective equipment; and occupational health. The practice scale included a series of 12 statements with the response options "Always", "Often", "Sometimes" or "Never". Practices were categorised as relating to the use of personal protective equipment, environmental control activities and administrative control activities.

The knowledge, attitudes and practices scales were formulated to include all levels of TB infection control: facility-level - on-site surveillance of TB among healthcare workers as well as IC plans; administrative control early recognition and management of TB; environmental control - includes adequate room ventilation; and personal protection measures - such as the use of surgical masks and N95 respirators [17]. The questionnaire was translated into the local language, SeSotho, and piloted at two facilities falling outside of the study area. Based on the findings of the pilot study, the questionnaire was revised and adapted. In addition, an observation schedule was developed to assess the actual implementation of TB infection control practices. The schedule was developed based on the WHO levels of TB infection control [17].
Data collection took place between September and November 2015. Researchers visited all 41 facilities and explained the research study to the facility managers, available nurses and facility-based CHCWs. The researchers completed an observation checklist of TB infection control measures. Subsequently the researchers distributed consent forms and the KAP questionnaires in envelopes to all nurses and CHCWs, who were asked to complete the questionnaires in their own time. Arrangements were made for the collection of completed questionnaires on specified dates. Follow-up telephone calls were also made to remind staff that the questionnaires would be collected. Upon collection of the questionnaires, they were edited and respondents were asked to fill out any missing information. While the questionnaires were anonymous, respondents could be traced through the consent forms, which could possibly have encouraged them to report more positive attitudes and better practices than was the case.

The data was captured, cleaned and analysed in IBM SPSS Statistics 23. Data was described using frequency counts and percentages for categorical variables and means and standard deviations for continuous variables. Composite scores were calculated in order to obtain the total knowledge, attitude and practice scores on each of the scales. On the knowledge scale, those respondents scoring $<65 \%$ were classified as having a poor level of knowledge, 65-79 \% was a moderate level of knowledge and $\geq 80 \%$ was a good level of knowledge. On the attitude scale, respondents scoring $80 \%$ and above were classified as having positive attitudes towards TB prevention and infection control. Respondents scoring $\geq 75 \%$ on the practice scale were classified as having good practices. The cut-off points for good levels of TB infection control knowledge and practices were informed by a similar study undertaken among healthcare workers in Northwest Ethiopia [36], where those answering $\geq 60 \%$ of knowledge questions correctly and $\geq 50 \%$ of practice questions correctly were considered to have good knowledge and practice, respectively.

The outcome variable was good TB prevention and infection control practices, and was defined as all respondents who correctly indicated that they would follow TB prevention and infection control practices $\geq 75 \%$ of the time. Pearson's $X^{2}$ test was used to establish any association between independent variables and the outcome variable. Binomial logistic regression analysis was used to determine which factors were significantly associated with good TB prevention and infection control practices. Independent variables included in the model were: sex (male/female), age, attitudes towards $\mathrm{TB}$, whether $\mathrm{TB}$ training was received (yes/no), job category $(\mathrm{CHCW} / \mathrm{as}-$ sistant or student nurse, professional nurse), location of PHC facility (Sub-districts A, B, C) and knowledge (poor, 
average, good). The odds ratios (ORs) together with their corresponding $95 \%$ confidence intervals (CIs) were estimated. The significance level considered for this study was 0.05 .

\section{Ethical considerations and authorisation}

Ethical clearance was obtained for the study from the Ethics Committee of the Faculty of Health Sciences, University of the Free State (ECUFS No92/2013), and the study was authorised by the Free State Department of Health. All participants completed a consent letter prior to participating in this research.

\section{Results}

\section{Demographic information}

A total of 34 out of 69 CHCWs (49.3\%) and 202 out of 404 nurses (50 \%) completed the questionnaires. The majority of respondents were female $(n=200 ; 87.7 \%)$, the average age was 44.19 years (standard deviation \pm 10.82 ) and they had been working in their current position for an average of 6.44 years (standard deviation \pm 8.19 ). Of the 236 respondents, 129 were professional nurses (54.7 \%), 73 assistant/student nurses (30.9\%) and $34 \mathrm{CHCWs}(14.4 \%)$. During the 12 months prior to the study, three-quarters of the respondents $(n=179$; $75.5 \%$ ) had received training on TB and HIV-related topics, including infection control, TB/HIV integration, IPT, and intensified case finding. More specifically, $57 \%$ $(n=130)$ had received training in TB infection control measures. Pearson's $X^{2}$ test was used to establish any association between the demographic variables and $\mathrm{TB}$ infection control practices. The only significant association was between sub-district and practices $(p<0.05)$ (Table 1).

\section{Knowledge of TB prevention and infection control}

Despite overall good scores on the knowledge scale, $42.8 \%(n=101)$ had an average score and $31.8 \%(n=75)$ had a good score, a closer examination of the individual items revealed the following problematic areas. Less than a quarter of the respondents knew the following correct answers: an HIV positive healthcare worker can still become ill with TB even if they practice infection control ( $n=49 ; 20.8 \%)$; TB patients with negative sputum smears are infectious $(n=53 ; 22.5 \%)$; and wearing a surgical mask does not protect healthcare workers from acquiring TB $(n=58 ; 24.6 \%)$. Only half of the respondents correctly knew that having a fever daily for more than a week was a symptom of TB $(n=189 ; 50.1 \%)$ and that an N95 respirator required a fit check $(n=119 ; 50.4 \%)$ - fit testing ensures better protection if the facial seal is correctly fitted [37]. Pearson's $X^{2}$ test revealed that there was a significant association between TB practices and levels of knowledge $(p<0.05)$ (Table 2).
Table 1 Demographic information

\begin{tabular}{lll}
\hline & $n=236(\%)$ & $p$-value \\
\hline Sex $^{\mathrm{a}}$ & $28(12.3)$ & \\
Male & $200(87.7)$ & .374 \\
Female & & \\
Sub-district & $84(35.6)$ & \\
A & $44(18.6)$ & $<.05$ \\
B & $108(45.8)$ & \\
C & & .134 \\
Highest formal education & $120(50.8)$ & \\
$\quad$ Secondary/matric & $116(49.2)$ & \\
Tertiary & & \\
Job title & $129(54.7)$ & .762 \\
Professional nurse & $73(30.9)$ & \\
Assistant/student nurse & $34(14.4)$ & \\
CHW & & \\
TB/HIV training in the past 12 months & \\
Yes & $179(77.5)$ & \\
No & $52(22.5)$ & \\
$a_{n}=228$ \\
${ }_{n} n=231$
\end{tabular}

\section{Attitudes}

Most respondent ( $n=189 ; 80.4 \%)$ had positive attitudes towards TB infection control practices. Despite these positive attitudes, the majority of respondents were afraid that they would acquire TB at work $(n=198$; $84.6 \%$ ); three in ten reported that their clinic was not concerned about their health and safety $(n=73 ; 31.4 \%)$ and did not provide adequate resources to prevent exposure to TB $(n=89 ; 38.2 \%)$. Most respondents $(n=205 ; 87.2 \%)$ had been screened for TB at work. TB screening for patients entails being asked a series of questions focusing on: cough for more than 2 weeks, weight loss, fever and night sweats. A positive response to one or more of these questions necessitates TB testing. Pearson's $X^{2}$ test revealed that there was a significant association between TB practices and attitudes $(p<0.05)$.

\section{Practices}

While good TB infection control practices were reported by $72.9 \%(n=161)$ of the respondents (see Table 3$)$, observations (see Table 4) at the facilities revealed this to not necessarily be the case. For example, environmental infection control practices were reportedly well implemented, with $95.2 \%(n=216)$ of the respondents indicating that they opened windows when possible to increase natural ventilation and $91.8 \%(n=213)$ explained to patients why it was important to keep windows open. Observations revealed that 12 (29.3\%) facilities had open windows in all consulting rooms and 
Table 2 Correct knowledge of TB prevention and infection control

\begin{tabular}{|c|c|}
\hline Correct responses to knowledge statements & $n(\%)$ \\
\hline \multicolumn{2}{|l|}{ TB symptoms } \\
\hline Prolonged cough is a symptom of TB (True) & $218(92.4)$ \\
\hline Unintentional weight loss is a symptom of TB (True) & $202(85.6)$ \\
\hline Fever every day for more than 1 week is a symptom of TB (True) & $189(50.1)$ \\
\hline Night sweats are a symptom of TB (True) & $231(97.9)$ \\
\hline \multicolumn{2}{|l|}{ TB transmission } \\
\hline TB patients with negative sputum smears can be considered infectious (False) & $53(22.5)$ \\
\hline TB can be spread through blood (False) & $210(89.0)$ \\
\hline Patients with TB commonly infect others by talking or singing (False) & $112(47.5 \%)$ \\
\hline \multicolumn{2}{|l|}{ Personal protective equipment } \\
\hline N95 respirators work just as well when wet or visibly dirty (False) & $189(80.1)$ \\
\hline Wearing a surgical mask can help healthcare workers protect themselves from TB (False) & $58(24.6)$ \\
\hline An N95 respirator provides an airtight seal on the face that the user does not need to check (False) & $119(50.4)$ \\
\hline \multicolumn{2}{|l|}{ Cough etiquette } \\
\hline Patients who are coughing should be given tissues or surgical masks to cover their mouths until TB has been excluded (True) & $210(89.0)$ \\
\hline If coughing/sneezing patients or suspects are using tissues or surgical masks, there no need for staff to wear N95 respirators (False) & $195(82.6)$ \\
\hline Before a TB suspect has a confirmed diagnosis, having him wear a surgical mask is unnecessary (False) & $168(71.2)$ \\
\hline \multicolumn{2}{|l|}{ Ventilation } \\
\hline Mechanical ventilation (like extractor fans) is always more effective than natural ventilation (open windows) for preventing TB (False) & $155(65.7)$ \\
\hline Open windows can help prevent the spread of TB (True) & $230(97.5)$ \\
\hline If a fan is used in a room, opening windows will not provide additional infection control (False) & $191(80.9)$ \\
\hline \multicolumn{2}{|l|}{ Separation of coughing patients } \\
\hline When entering the clinic, every patient should be asked if they are coughing (True) & $218(92.4)$ \\
\hline Keeping coughing and non-coughing patients apart in the clinic will help to stop TB from spreading (True) & $186(78.8)$ \\
\hline TB suspects in the waiting area should not wait just as long as everyone else, and should not be rushed through the queue (False) & $200(84.7)$ \\
\hline \multicolumn{2}{|l|}{ TB/HIV } \\
\hline An HIV positive staff member cancan get sick with TB if they practice TB prevention strategies (False) & $49(20.8)$ \\
\hline HIV positive staff who are healthy and on ARVs should still try to avoid working in high risk areas (True) & $158(66.9)$ \\
\hline An HIV positive person is more likely than an HIV negative person to become sick with TB if exposed to TB (True) & $218(92.4)$ \\
\hline
\end{tabular}

9 (22.5\%) facilities had open windows in all waiting areas. This was despite the observation that most facilities $(n=35 ; 85.4 \%)$ had "open window" stickers pasted to their windows to remind them to open windows to prevent TB transmission.

Poor practices were reported by the respondents when it came to using personal protective equipment: only $52.2 \% \quad(n=106)$ always wore a N95 respirator when collecting sputum from a patient presumed to have TB and 15.4\% never used a N95 respirator in the TB consultation room. Observations at the facilities found supporting evidence for poor personal protective practices, with only five (12.2\%) facilities having TB nurses wearing N95 respirators, despite N95 respirators being available at 32 facilities (78.0\%).
Self-reported administrative controls that were poorly implemented included not separating (presumptive) TB patients $(n=100 ; 46.9 \%)$ from non-coughing patients. This was supported by the observation that only 11 facilities $(26.8 \%)$ had separate waiting areas for (presumptive) TB patients. Furthermore, while approximately one- third of the respondents $(n=71 ; 32.9 \%)$ did not always give coughing patients tissues or surgical masks to cover their cough, the reality was that patients at only two clinics were observed to be wearing such masks. In addition, only 7 (17.1\%) clinics had tissues available for coughing patients and only three $(7.3 \%)$ had face masks.

\section{Predictors of good practice}

A binomial logistic regression was performed to ascertain the effects of age, TB infection control knowledge 
Table 3 Self-reported TB infection control practices

\begin{tabular}{|c|c|c|c|c|}
\hline & $\begin{array}{l}\text { Always } \\
n(\%)\end{array}$ & $\begin{array}{l}\text { Often } \\
n(\%)\end{array}$ & $\begin{array}{l}\text { Sometimes } \\
n(\%)\end{array}$ & $\begin{array}{l}\text { Never } \\
n(\%)\end{array}$ \\
\hline \multicolumn{5}{|l|}{ Personal Protective Equipment } \\
\hline I use an N95 respirator when collecting sputum from a patient & $106(52.2)$ & $27(13.3)$ & $44(21.7)$ & $26(12.8)$ \\
\hline I use an N95 respirator in the TB consultation room & $104(50.0)$ & $35(16.8)$ & $37(17.8)$ & $32(15.4)$ \\
\hline I interact with patients while using a N95 respirator & $111(50.5)$ & $30(13.6)$ & $49(22.3)$ & $30(13.6)$ \\
\hline \multicolumn{5}{|l|}{ Environmental } \\
\hline I open windows when possible to increase natural ventilation & $216(95.2)$ & $10(4.4)$ & & $1(0.4)$ \\
\hline I explain to patients why it is important to keep windows open & $213(91.8)$ & $13(5.6)$ & $5(2.2)$ & $1(0.4)$ \\
\hline I may turn off fans if they become too noisy or cause cold air to blow around & $25(10.7)$ & $25(10.7)$ & $55(23.6)$ & 77 (32.6) \\
\hline \multicolumn{5}{|l|}{ Administrative } \\
\hline I order sputum specimens when I suspect TB & $209(95.2)$ & $6(2.7)$ & $7(3.1)$ & $4(1.8)$ \\
\hline Patients with suspected TB are isolated from other patients & $107(49.1)$ & $24(11.0)$ & $39(17.9)$ & $48(22.0)$ \\
\hline I ask each patient who enters the clinic if they are coughing and for how long & $205(88.7)$ & $10(4.3)$ & $12(5.2)$ & $4(1.7)$ \\
\hline I move TB suspects to a separate waiting area & $113(53.1)$ & $28(13.1)$ & $28(13.1)$ & $44(20.7)$ \\
\hline $\begin{array}{l}\text { I move TB suspects to the front of the queue to minimise the amount of time } \\
\text { that they spend in the waiting area around other patients }\end{array}$ & $159(70.9)$ & $27(12.3)$ & $22(10.0)$ & $15(6.8)$ \\
\hline I give tissues/surgical masks to coughing patients & $145(67.1)$ & $22(10.2)$ & $30(13.9)$ & $19(8.8)$ \\
\hline
\end{tabular}

and attitudes, TB/HIV training, job category and PHC facility location on good TB infection control practices. Linearity of the continuous variables with respect to the logit of the dependent variable was assessed via the BoxTidwell procedure. Based on this assessment, all continuous independent variables were found to be linearly related to the logit of the dependent variable. There were six outliers, but no high leverage or influential values.

Table 4 TB infection control measures observed at facilities

\begin{tabular}{ll}
\hline Observed & $n(\%)$ \\
\hline Personal protective equipment & $32(78.0)$ \\
N95 respirators available & $5(12.2)$ \\
TB nurses wearing N95 respirators & \\
Environmental controls & $35(85.4)$ \\
Open window stickers & $12(30.0)$ \\
Open window register & $12(29.3)$ \\
Open windows in all consulting rooms & $9(22.5)$ \\
Open windows in all waiting areas & \\
Administrative controls & $7(17.1)$ \\
Tissues available for coughing patients & $3(7.3)$ \\
Face masks available for coughing patients & $2(4.9)$ \\
Coughing patients wearing masks & $3(7.3)$ \\
Appropriate colour -coded waste bins available in & \\
waiting area for tissues/masks & $37(92.5)$ \\
Appropriate colour -coded waste bins available in & \\
consulting rooms for tissues/masks & $11(26.8)$ \\
\hline
\end{tabular}

Since the unusual points were not due to data entry errors, it was decided to keep them in the analysis. The logistic regression model was statistically significant, $\chi 2$ $(9)=29.821, p<0.001$. The model explained $19.7 \%$ (Nagelkerke R2) of the variance in the tendency to have good TB infection control practices and correctly classified $74.9 \%$ of cases. Sensitivity was $92.5 \%$ and specificity was $29.8 \%$, the positive predictive value was $77.1 \%$ and the negative predictive value was $39.3 \%$. Attitudes and high levels of knowledge were statistically significantly associated with good TB practices after controlling for all other variables in the model. For every unit increase in attitudes, good practices increased 1.090 times (CI:1.016-1.169). Respondents with high levels of knowledge ( $\geq 80 \%$ ) were 4.029 (CI: $1.550-10.469$ ) times more likely to have good practices when compared to respondents with poor levels of knowledge (<65\%). Although only borderline significant $(p=0.05)$, respondents working in facilities in Sub-district B were 3.024 times more likely to have good TB infection control practices than respondents working in Sub-district A (Table 5).

\section{Discussion}

The increasing evidence of healthcare facility acquired TB in both patients and healthcare workers in South Africa, has led to a greater interest in TB infection control $[1,3,5,21,38]$. International and national policies $[10,11]$ recommend several simple and effective infection control measures aimed at reducing the nosocomial spread of TB in healthcare settings, particularly in resource poor settings. However, as in most endemic 
Table 5 Binomial logistic regression analysis of factors associated with good TB prevention and infection control practices

\begin{tabular}{|c|c|c|c|c|}
\hline Variables & $n(\%)$ & Odds Ratio & $95 \% \mathrm{Cl}$ & $P$ value \\
\hline Age (mean and std deviation) & $44.9(10.783)$ & 1.031 & $0.997-1.067$ & .078 \\
\hline Attitudes (mean and std deviation) & $58.45(6.045)$ & 1.090 & $1.016-1.169$ & .016 \\
\hline \multicolumn{5}{|l|}{ Training } \\
\hline Received no training (ref) & $39(24.8)$ & 1 & & \\
\hline Received training & $118(75.2)$ & 0.572 & $0.232-1.411$ & .225 \\
\hline \multicolumn{5}{|l|}{ Job category } \\
\hline CHW (ref) & $24(14.9)$ & 1 & & \\
\hline Assistant/student nurse & $50(31.1)$ & 0.341 & $0.102-1.141$ & .081 \\
\hline Professional nurse & $87(54.0)$ & 0.3725 & $0.107-1.291$ & .119 \\
\hline \multicolumn{5}{|l|}{ Location of PHC facility } \\
\hline A & $48(29.8)$ & 1 & & \\
\hline B & $34(21.1)$ & 3.024 & $0.998-9.162$ & .050 \\
\hline C & $79(49.1)$ & 1.778 & $0.813-3.887$ & .150 \\
\hline \multicolumn{5}{|l|}{ Knowledge } \\
\hline Poor (ref) & $31(19.3)$ & 1 & & \\
\hline Average & $72(44.7)$ & 1.867 & $0.822-4.242$ & .136 \\
\hline Good & $58(36.0)$ & 4.029 & $1.550-10.469$ & .004 \\
\hline
\end{tabular}

settings, healthcare workers in South Africa fail to implement appropriate infection control measures to protect both themselves and their patients from TB infection [19-27, 30]. Our study aligns with these findings, and identified that almost three in ten healthcare workers failed to implement good TB infection control practices in their facilities. As this is based on selfreported compliance, the real situation may be even worse in the facilities. Respondents tend to present themselves in a favourable light (i.e. social desirability bias) [39], and we attempted to counter this by undertaking a number of observations at the facilities, which indeed did reveal poorer practices.

Poor practices relating to administrative TB infection controls were recorded. In particular, and as established in similar research undertaken in South African health facilities $[19,21,23,27,33]$, we found that approximately half of the respondents always isolated patients with presumptive TB or moved these patients to a separate waiting area. Furthermore, only a quarter of the facilities had separate waiting areas for TB patients. In spite of these poor practices, the majority of respondents knew that separating coughing and non-coughing patients in the facility would help to prevent the spread of TB. A further concern was the inadequate provision of tissues and masks to coughing patients [23, 27], which was supported by observations at the facilities - only two facilities had patients wearing masks on the days of the fieldwork visits. This was the case, despite the majority of respondents reporting that coughing patients should be given masks or tissues to cover their cough. This lack of attention to the implementation of administrative control measures is a serious concern given that these are the first line of defence in healthcare facilities [17] to prevent patient and healthcare worker exposure to TB. Furthermore, these measures are simple and not costly to implement.

Environmental infection control practices were reportedly better implemented than administrative measures and the use of personal protective equipment. The majority of respondents indicated that they opened windows when possible to increase natural ventilation and explained to patients why it was important to keep windows open. However, in practice, and in line with previous research [22, 23, 27, 33, 40], observations at the facilities revealed that only two in ten had all windows open in consultation rooms and waiting areas on the day of fieldwork visits. This was despite the fact that the research was undertaken in the spring, and an even worse picture could present in the winter.

While personal protective measures (i.e. the use of respiratory protection) are the last step in the hierarchy of infection control [17], N95 respirators can filter out TB bacilli and are recommended for use in TB control [41]. Despite these recommendations, poor knowledge and practices were reported when it came to the use of personal protective equipment. This was also found to be the case in other TB infection control studies conducted in South Africa [19, 21, 23, 27, 40, 42]. Moreover, on the days of the fieldwork visits, TB nurses at only five of facilities were wearing N95 respirators. 
Overall, respondents had fairly good levels of knowledge about TB infection control, with the majority scoring above $65 \%$ on the scale (c.f. $[19,36,43])$. The main areas of concern related to the infectiousness of patients, use of personal protective equipment, and co-infection with HIV. Attitudes towards infection control were also overwhelmingly positive. In this regard, it was not surprising to find that attitudes and good levels of infection control knowledge were statistically significant predictors of good practices. While other research has also identified knowledge to be a predictor of good practices [36], this was not entirely the case in an Ethiopian study, where TB-related training, and not knowledge, was found to be a predictor of good practices. However, they did find positive correlations between knowledge and practice [43]. To the contrary, our study did not find $\mathrm{TB} / \mathrm{HIV}$-related training to be a predictor of good practices. This is supported by Temesgen and Demissie [36] who also found that training on TB infection control did not have an influence on practice. This raises questions regarding the type and quality of training that was provided as it has implications for strategies to improve TB infection control practices. It is possible that the training was too theoretical rather than skills-based. It is important to not only train but also support/mentor healthcare workers on skills to strengthen the implementation of TB infection control strategies $[36,43]$.

A limitation of the study is the low response rate. The overall response rate of $50 \%$ is low - despite numerous follow up visits to the facilities - but not much different from a similar study [27]. It is well established that healthcare worker participation in selfadministered surveys is generally low [27, 44]. Possible explanations for the low response rate include overworked facility staff, large patient numbers and a lack of incentives to complete the questionnaire. Further limitations of the study include self-reports of good practices, although countered to a degree by observations at the facilities, as well as the research taking place in spring, which could also have impacted on practices such as open windows. More robust claims can be facilitated with a longitudinal design, which could also add to the further exploration of causal mechanisms tied to good infection control practices. For example, this study did not investigate the role of patients in ensuring TB infection control in healthcare facilities. Previous research has found that knowledge and acceptability of patient related infection control measures is key to patient adherence not only in healthcare facilities but also in household settings and the community [45].

\section{Conclusion}

Previous research on TB infection control in South Africa mainly focused on hospital settings. The current study addresses this gap by describing practices in PHC facilities (the main entry point for TB patient-care) and exploring factors that promote good infection control practices among healthcare workers. Furthermore, multiple methods were used (self-administered questionnaires and structured observations) to counter the problem of social desirability bias associated with self-reports so as to obtain a more accurate picture of the state of implementation of TB infection control in PHC facilities.

In conclusion, positive attitudes and good levels of knowledge regarding TB infection control were the main factors associated with good infection control practices. Although many respondents reported good infection control practices - which was somewhat countered by the observations - there are areas that require attention, particularly those related to administrative controls and the use of personal protective equipment. Improving infection control practices relies on modifying healthcare worker behaviour. The application of sophisticated behavioural science methods to TB infection control research could lead to better implementation of practices [46]. Conventional training does not appear to improve TB infection control practices, new strategies are required to improve TB infection control in PHC facilities.

\section{Abbreviations \\ ART: Antiretroviral treatment; CHCWs: Community healthcare workers; \\ HAls: Healthcare associated infections; HIV: Human immunodeficiency virus; IPT: Isoniazid preventative therapy; MDR: Multi-drug resistant; ORs: Odds ratios; PHC: Primary health care; TB: Mycobacterium tuberculosis; WHO: World health organization; XDR: Extensively drug resistant}

\section{Acknowledgments}

The assistance of the Free State Department of Health, project fieldworkers, participating healthcare workers, and Quantemna Quantitative Research Specialists are gratefully acknowledged.

\section{Funding}

This work is based on the research supported in part by the National Research Foundation of South Africa for the Grant 87696. Any opinion, finding and conclusion or recommendation expressed in this material is that of the author (s) and the NRF does not accept any liability in this regard.

\section{Availability of data and materials}

The dataset analysed during the current study is available from the corresponding author on reasonable request.

\section{Authors' contributions}

$M E, A j v R$ and GK designed the research, constructed the instruments and conducted the fieldwork. ME conducted the data analysis and drafted the manuscript. AjvR, GK and HCJvR contributed to the manuscript. All authors read and approved the manuscript.

\section{Authors' information}

ME, AjvR, GK, HCJvR: Centre for Health Systems Research \& Development, University of the Free State, Bloemfontein, South Africa; AjvR: Health and Demographic Research Unit, Department of Sociology, Ghent University, Ghent, Belgium, and Department of Political Science, Stellenbosch University, Stellenbosch, South Africa.

Competing interests

The authors declare that they have no competing interests. 


\section{Consent for publication}

Not applicable.

\section{Ethics approval and consent to participate}

Ethical clearance granted by the Ethics Committee of the Faculty of Health Sciences, University of the Free State (ECUFS No92/2013).

\section{Author details}

${ }^{1}$ Centre for Health Systems Research \& Development, University of the Free State, Nelson Mandela Road, Bloemfontein 9300, South Africa. ${ }^{2}$ Health and Demographic Research Unit, Department of Sociology, Ghent University, Korte Meer 5, Ghent 9000, Belgium. ${ }^{3}$ Department of Political Science, Stellenbosch University, Corner Merriman and Ryneveld Street, Stellenbosch 7602, South Africa

\section{Received: 13 July 2016 Accepted: 27 October 2016}

Published online: 04 November 2016

\section{References}

1. Harries AD, Zachariah R, Tayler-Smith K, Schouten EJ, Chimbwandira F, Van Damme $W$, et al. Keeping health facilities safe: one way of strengthening the interaction between disease-specific programmes and health systems. Trop Med Int Health. 2010;15(12):1407-12.

2. WHO. Health care-associated infections Fact Sheet. World Health Organization. 2016. http://www.who.int/gpsc/country_work/gpsc_ccisc_ fact_sheet_en.pdf. Accessed 6 Apr 2016.

3. Pillay M, Sturm AW. Noscomial transmission of F15/LAM4/KZN genotype of Mycobacterium tuberculosis in patients on tuberculosis treatment. Int J Tuberc Lung Dis. 2010;14:223-30

4. WHO. Global tuberculosis report 2013. Geneva: World Health Organizaiton; 2013.

5. Churchyard GJ, Mametja LD, Mvusi L, Ndjeka N, Hesseling AC, Reid A, et al. Tuberculosis control in South Africa: Successes, challenges and recommendations. SA Med J. 2014;104 Suppl 1:244-8.

6. WHO. Global tuberculosis report 2015. Geneva: World Health Organizaiton; 2015

7. NDoH. National antenatal sentinel HIV \& syphilis prevalence survey in South Africa, 2012. Pretoria: National Department of Health; 2013.

8. Gandhi NR, Moll A, Sturm AW, Pawinski R, Govender T, Lalloo U, et al. Extensively drug-resistant tuberculosis as a cause of death in patients coinfected with tuberculosis and HIV in a rural area of South Africa. Lancet. 2006;368:1575-80

9. NDoH. National Tuberculosis Management Guidelines: Pretoria: National Department of Health. 2014.

10. Joshi R, Reigold AL, Menzie D, Pai M. Tuberculosis among Health-Care Workers in Low- and Middle-Income Countries: A Systematic Review. PLoS Med. 2006:3(12), e494.

11. URC South Africa. Tuberculosis in Healthcare Workers: Findings from South Africa. University Research Co. LLC \& Desmond Tutu Tuberculsosis Centre. 2013.

12. Baussano I, Nunn P, Williams B, Pivetta E, Bugiani M, Scano F. Tuberculosis among Health Care Workers. Emerg Infect Dis. 2011;17(3):488-94.

13. McCarthy KM, Scott LE, Gous N, Tellie M, Venter WDF, Stevens WS, et al. High incidence of latent tuberculous infection among South African health workers: an urgent call for action. Int J Tuberc Lung Dis. 2015;19(6):647-53.

14. Adams S, Ehrlich R, Baatjies R, Van Zyl-Smit RN, Said-Hartley Q, Dawson R, Dheda K. Incidence of occupational latent tuberculosis infection in South African healthcare workers. Eur Respir J. 2015:45:1364-73.

15. Adamsi S, Morar R, Kolbe-Alexander T, Jeebhay MF. Occupational Health Challenges Facing the Department of Health. In: Protecting employees against tuberculosis and caring for former mineworkers with occupational health disease, in South African Health Review 2012/13 Padarath A, English R, Editors. Durban: Health Systems Trust; 2013. p. 67-82.

16. Von Delft A, Dramowski A, Khosa C, Kotze K, Lederer P, Mosidi T, et al. Why healthcare workers are sick of TB. Int J Infect Dis. 2015:32:147-51.

17. WHO. WHO policy on TB infection control in health-care facilities, congregate settings and households 2009. Geneva: World Health Organization; 2009

18. NDoH. The draft national infection prevention and control guidelines for TB, MDR TB and XDR TB. Pretoria: National Department of Health; 2007.
19. Kanjee Z, Catterick K, Moll AP, Amico KR, Friedland GH. Tuberculosis infection control in rural South Africa: survey of knowledge, attitude and practice in hospital staff. J Hosp Infect. 2011;79:333-8.

20. Kanjee Z, Amico KR, Li F, Mbolekwa K, Moll AP, Friedland GH. Tuberculosis infection control in a high drug-resistance setting in rural South Africa: Information, motivation, and behavioural skills. J Infect Public Health. 2012:5:67-81.

21. Farley JE, Tudor C, Mphahlele M, Franz K, Perrin NA, Dorman S, et al. A national infection control evaluation of drug-resistant tuberculosis in hospitals in South Africa. Int J Tuberc Lung Dis. 2012;16(1):82-9.

22. Naidoo S, Seevnarain K, Nordstrom DL. Tuberculosis infection control in primary health clinics in eThekwini, KwaZulu-Natal, South Africa. Int J Tuberc Lung Dis. 2012;16(12):1600-4

23. Engelbrecht MC, Janse Van Rensburg A. TB infection control practices in PHC facilities in three districts of South Africa. SA J Epi Infect. 2013;287(4): 221-6.

24. Zelnick JR, Gibbs A, Loveday M, Padayatchi N, O'Donnell MR. Health-care workers' perspectives on workplace safety, infection control, and drugresistant tuberculosis in a high-burden HIV setting. J Public Health Policy. 2013:34(3):388-402.

25. Bhebhe LT, Van Rooyen C, Steinberg WJ. Attitudes, knowledge and practices of healthcare workers regarding occupational exposure of pulmonary tuberculosis. Afr J Prim Health Care Fam Med. 2014;6(1):1-6.

26. Engelbrecht MC, Yassi A, Spiegel JM, Van Rensburg AJ, O'Hara LM, Bryce EA, et al. Tuberculosis and blood-borne infectious diseases: Workplace conditions and practices of healthcare workers at three public hospitals in the Free State. South Afr J Epidemiol Infect. 2015:30(1):23-8.

27. Malangu N, Mngomezulu M. Evaluation of tuberculosis infection control measures implemented at primary health care facilities in Kwazulu-Natal province of South Africa. BMC Infect Dis. 2015;15:117.

28. Yes!Media. Local Government Handbook. 2016. http://www. localgovernment.co.za/provinces/view/2/free-state. Accessed 8 July 2016.

29. Day C, Gray A. Health and related indicators. In: Padarath A, English R, editors, South African health review 2013/14. Durban: Health Systems Trust; 2014.

30. Janse van Rensburg A, Engelbrecht MC, Spiegel JM, O'Hara LM, Bryce EA, Nophale LE, et al. Occupational health and safety of nurses in public hospitals in the Free State, South Africa. Occ Health S Africa. 2016:22(2):8-14.

31. Corbett EL. Health worker access to HIV/TB prevention, treatment and care services in Africa: situational analysis and mapping of routine and current best practices. London: London School of Hygiene \& Tropical Medicine; WHO/HIV Department; WHO/TB Department; Global Health Workforce Alliance; 2007.

32. Parmeggiani $C$, Abbate R, Marinelli P, Angelillo IF. Healthcare workers and health care-associated infections: knowledge, attitudes, and behavior in emergency departments in Italy. BMC Infect Dis. 2010;10(1):35.

33. Mphahlele MT, Tudor C, Van der Walt M, Farley J. An infection control audit in 10 primary health-care facilities in the Western Cape Province of South Africa. Int J Infect Control. 2012:8:3.

34. Tenna A, Stenehjem EA, Margoles L, Kacha E, Blumberg HM, Kempker RR. Infection control knowledge, attitudes, and practices among healthcare workers in Addis Ababa, Ethiopia. Infect Control Hosp Epidemiol. 2013; 34(12):128-1296

35. Nicol L, Mehtar S, Dheda K, Adams S, Van der Walt M, Osman M. A systematic review of the epidemiology of and programmatic response to TB in health care workers in South Africa. Evidence to Inform South African Tuberculosis policies (EVISAT) Project. Geneva: World Health Organization; 2014.

36. Temesgen C, Demissie M. Knowledge and practice of tuberculosis infection control among health professionals in Northwest Ethiopia. BMC Health Serv Res. 2014;14:593.

37. CDC 2016. The National Personal Protective Technology Laboratory (NPPTL): Ancillary Respirator Information. http://www.cdc.gov/niosh/npptl/topics/ respirators/disp_part/respsource3fittest.html. Accessed 22 Sept 2016.

38. O'Donnell MR, Jarand J, Loveday M, et al. High incidence of hospital admissions with multidrug-resistant and extensively drug-resistant tuberculosis among South African health care workers. Ann Intern Med. 2010;153:516-22.

39. Babbie E. The basics of social research (5th ed). Belmont: Wadsworth: 2011.

40. Sissolak D, Bamford CM, Mehtar S. The potential to transmit mycobacterium tuberculosis at a South African tertiary teaching hospital. Int J Infect Dis. 2010;14:e423-8.

41. WHO Guidelines for the prevention of tuberculosis in health care facilities in resource-limited settings. Geneva: World Health Organization; 1999. 
42. URC. Tuberculosis in healthcare workers: findings from South Africa. Pretoria: University Research Co; 2012. www.urc-chs.com/resources/tuberculosishealthcare-workers-findings-south-africa. Accessed 3 Sept 2012.

43. Gizaw GD, Alemu ZA, Kibret KT. Assessment of knowledge and practice of health workers towards tuberculosis infection control and associated factors in public health facilities of Addis Ababa, Ethiopia: A cross-sectional study. Arch Public Health. 2015;73:15.

44. Cook JV, Dickinson HO, Eccles MP. Response rates in postal surveys of healthcare professionals between 1996 and 2005: An observational study. BMC Health Serv Res. 2009;9:160.

45. Gonzalez-Angulo Y, Geldenhuys H, Van As D, Buckerfield N, Shea S, Mahomed H, Hanekom W, Hatherill M. Knowledge and acceptability of patient-specific infection control measures for pulmonary tuberculosis. Am J Infect Control. 2013;14(8):717-22.

46. Pittet D. The Lowbury lecture: behaviour in infection control. J Hosp Infect. 2004;58:1-13.

\section{Submit your next manuscript to BioMed Central} and we will help you at every step:

- We accept pre-submission inquiries

- Our selector tool helps you to find the most relevant journal

- We provide round the clock customer support

- Convenient online submission

- Thorough peer review

- Inclusion in PubMed and all major indexing services

- Maximum visibility for your research

Submit your manuscript at www.biomedcentral.com/submit 